\title{
Soberanía alimentaria, sustentabilidad y cambio climático La soberanía alimentaria ¿Una alternativa ante el acelerado cambio climático?
}

Ronnie Lizano

\section{SciELO Books / SciELO Livros / SciELO Libros}

LIZANO, R. La soberanía alimentaria ¿Una alternativa ante el acelerado cambio climático?. In: ESTEBAN, D., ARTACKER, T., and LIZANO, R., coord. Cambio climático, biodiversidad y sistemas agroalimentarios: avances y retos a 10 años de la Ley Orgánica del Régimen de la Soberanía Alimentaria en Ecuador [online]. Quito: Editorial Abya-Yala, 2020, pp. 219-231. ISBN: 978-9978-10-466-8.

http://doi.org/10.7476/9789978105689.0013.

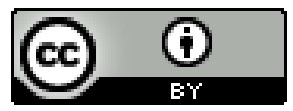

All the contents of this work, except where otherwise noted, is licensed under a Creative Commons Attribution 4.0 International license.

Todo o conteúdo deste trabalho, exceto quando houver ressalva, é publicado sob a licença Creative Commons Atribição 4.0.

Todo el contenido de esta obra, excepto donde se indique lo contrario, está bajo licencia de la licencia Creative Commons Reconocimento 4.0. 


\section{La soberanía alimentaria ¿Una alternativa ante el acelerado cambio climático?}

Ronnie Lizano $^{1}$

\section{Cambio climático y agronegocio}

A nivel global, el $97 \%$ de emisiones de $\mathrm{CO}_{2}$ lo generan los países "desarrollados", y al parecer no están dispuestos a reducirlas ya que en este mundo globalizado los mercados son el centro de atención y la prioridad. El modelo de desarrollo de muchos países occidentales se ha exportado como una receta para salir de la pobreza, desde esta visión hegemónica, América Latina ha sido considerada como una economía emergente y la agricultura no ha escapado al concepto de globalización y estandarización debido al modelo de revolución verde ${ }^{2}$ que ha imperado en las últimas décadas con promesas como paliar el hambre a nivel mundial. Sin embargo, ha tenido consecuencias nefastas en muchos contextos generando incluso la

1 Máster en Desarrollo Rural y gestión sustentable en la Universidad Politécnica de Madrid y doctorando en la Universidad Andina Simón Bolívar, Quito. Coordinador del Grupo de Investigación en Ciencias Ambientales-GRICAM. Línea de investigación en agroecología, ambiente y territorio. Docente en la Universidad Politécnica Salesiana.

2 La revolución verde hace referencia al modelo de agricultura exportada por los Estados Unidos, que se basa en el uso intensivo de agrotóxicos, fertilizantes sintéticos, semilla híbrida y maquinaria agrícola. 
enajenación de los modos de producción de los campesinos, deterioro de su salud y migración a las ciudades que en muchos casos llegan a engrosar los cinturones de miseria. En este contexto, la apuesta por la soberanía alimentaria y el movimiento por la agroecología brindan oportunidades a los campesinos para trabajar en una lógica de la vida, de la biodiversidad, donde las fincas agroecológicas se pueden mirar como espacios donde la familia campesina busca dignificarse y reivindicar su derecho al trabajo y a un ambiente saludable.

El cambio climático afecta seriamente la agricultura porque conlleva a la alteración de los regímenes de lluvia y temperatura, además de fenómenos climáticos que experimentarán más frecuente e intensamente los países en vía de desarrollo, lo cual puede comprometer la soberanía alimentaria. Se presentan dos visiones claramente marcadas, el discurso de la seguridad alimentaria, funcional al modelo agrícola dominante, y por el otro, la alternativa de la agroecología y la búsqueda de la soberanía alimentaria; dos paradigmas en disputa, tanto del territorio, del espacio ecológico y de los procesos salud-enfermedad.

En la agricultura, la revolución verde impone condicionamientos, por ejemplo, al introducir como "factores de innovación tecnológica" lo que en realidad es un "modo de imposición tecnológica" que perpetúa la dependencia de los agricultores hacia los insumos: fertilizantes, pesticidas, maquinaria, semillas híbridas y actualmente semillas transgénicas. La estrategia de la revolución verde funcionó bien en áreas dotadas de un clima estable y energía barata. $\mathrm{Al}$ entrar en la segunda década del siglo XXI, este modelo agrícola industrial se tornó inviable, ya que los insumos, equipos y combustibles fósiles, de los cuales depende, empezaron a agotarse y a encarecerse. A medida que el cambio climático se intensifica, estos sistemas agrícolas intensivos se vuelven menos resistentes y más vulnerables. De acuerdo con Díaz (2013), en la búsqueda de posibles adaptaciones agrícolas al cambio climático se han desarrollado modificaciones genéticas de organismos para que los cultivos produzcan bajo condiciones estresantes, se proponen los biocombustibles y la economía verde, lo cual básicamente consiste en perpetuar la revolución verde. 
La agricultura industrial contribuye marcadamente al cambio climático mediante la emisión de gases de efecto invernadero, principalmente el óxido nitroso (N2O) y el dióxido de carbono (CO2) por el consumo de combustibles fósiles utilizados en la mecanización agrícola y en la irrigación, en los fertilizantes y pesticidas químicos y en el transporte de los productos agrícolas, en las extendidas cadenas verticales de la agroindustria y los agronegocios internacionales (Díaz, 2013). Globalmente, la producción de alimentos es responsable del 21\% de las emisiones de gases de efecto invernadero (IPCC, 2014; FAO, 2015). De acuerdo a la FAO, las emisiones generadas por la aplicación de fertilizantes sintéticos en 2012 aportaron de un 14\% al 21\% total generado por el modelo agrícola actual (FAO, 2015). Un peso especial en el aporte al cambio climático tiene la producción de carne, la producción de alimento animal ocupa un $40 \%$ de la producción agrícola mundial (FAO, 2012). La FAO estima que un promedio de $36 \%$ de la producción de cereal del mundo es usado para la alimentación del ganado de leche y carne (FAO, 2012). Esto se vuelve muy contradictorio ya que, frente a más de 1000 millones de personas con hambre en el mundo, la gran cantidad de los cereales se destinan a la alimentación de animales, y esta gran demanda ha hecho que se intensifique el sistema de producción pecuario a un punto en que la gran mayoría de ganado de leche y carne de nivel industrial son alimentados con cereales, como si se tratase de un animal monogástrico, provocando trastornos fisiológicos en los rumiantes. ${ }^{3}$ Además, el uso de nuevas áreas para pastos para ganadería es una de las principales causas de deforestación en América Latina. En los últimos 50 años, el consumo de carne se ha cuadruplicado: 45 millones de toneladas en 1950; 300 millones de toneladas en 2018; y 500 millones de toneladas en 2050. En promedio cada norteamericano consume 80,6 kg de carne por año. De acuerdo a la OMS, una reducción a $25 \mathrm{~kg}$ sería una victoria para nuestra salud y para el planeta (FAO, 2011).

3 Los rumiantes son animales que tienen cuatro cavidades gástricas o estómagos donde es procesado el pasto que ingieren a través de microorganismos y de esta forma asimilan los nutrientes necesarios para su alimentación. 
- De acuerdo con la OMS, la cantidad ideal de carne para una dieta óptima es de 500 gramos por semana, $2 \mathrm{~kg}$ por mes, o $24 \mathrm{~kg}$ por año.

- Sobre el 95\% de la carne que consumimos viene de fincas industriales que son colectivamente responsable del 14.5\% de las emisiones de gases de efecto invernadero (Gerber et al. 2013)

- Producir $1 \mathrm{~kg}$ de carne genera $36,4 \mathrm{~kg}$ de $\mathrm{CO}_{2}$, equivalente a las emisiones de un carro en un viaje de $250 \mathrm{~km}$; y utiliza no menos de 15000 litros de agua (Fanelli, 2007).

Las cadenas verticales de la agroindustria representan uno de los aspectos más problemáticos, debido a la cantidad de eslabones entre los productores y consumidores; mientras mayor es la cantidad de eslabones en la cadena, más distancia existe entre agricultores y consumidores, y esta distancia se expresa en un colosal consumo de energía para producir, procesar y transportar estos alimentos. Esta comida que se muestra más barata en el mercado, pero a costa del deterioro social, económico y ambiental.

El modelo alimentario estadounidense que se trasladó a los países subdesarrollados mediante una relación centro-periferia, incluyó el dominio de las empresas transnacionales, la extensión de la revolución verde en sus diversos componentes, así como, la utilización política y económica de la ayuda alimentaria, que en no pocos casos contribuyó a desestimular la producción local de alimentos y su sustitución por productos foráneos importados (George, 1981).

Lo que se cuestiona de la revolución verde, es ser un modelo de imposición tecnológica, un modelo que artificializa los hábitats naturales, donde el monocultivo reemplaza la biodiversidad, donde se pone como centro solamente la productividad, aunque tenga costos ocultos a nivel social y ambiental. De la mano del modelo de la revolución verde que reproduce la lógica de la estandarización a través de monocultivos, está atado el proceso de acaparamiento de tierras, que es otro fenómeno global, que es impulsado por una es- 
trategia espacial de dominio de capital, lo cual, como Rubio (2015) afirma, ha traído consigo que germine la dimensión territorial de la crisis capitalista. El autor ha denunciado 400 casos de acaparamiento de tierras sobre una superficie de 35 millones de hectáreas en 66 países del mundo, reflejando lo que manifiesta al decir que el norte pierde competitividad y busca soluciones espaciales a su decadencia en el sur. Como señala Rubio (2015), las tierras se compran en países del sur, con buena calidad de suelo, abundancia de recursos naturales y de agua, y con fuerza de trabajo barata. Esta solución espacial, representa un intento a corto plazo para resolver las contradicciones de los crecientes gastos agroindustriales y los crecientes gastos de reproducción de la fuerza de trabajo, lo que según McMichael (2012), agudizará las contradicciones ecológicas y sociales.

Las consecuencias de este proceso que por lo general lleva a implantar monocultivos para la exportación, entre ellos los agrocombustibles, han sido muy graves para los pobladores rurales, quienes en muchas ocasiones sufren el despojo o la venta forzada de sus parcelas (Rubio, 2010); acompaña a esto la degradación de las tierras y de los recursos naturales amenazando la agricultura familiar campesina en muchos países. Esta es la urgencia ante la crisis civilizatoria que está viviendo nuestro planeta, un deterioro acelerado de la sociedad rural y su soberanía alimentaria. Para Houtart (2016), la historia nos ha enseñado que es muy difícil reconstruir una agricultura campesina cuando ya ha sido eliminada, este es el caso de Venezuela donde la agricultura fue destruida por la renta petrolera, y de Cuba, que sufrió siglos de monocultivos de azúcar.

Las consecuencias de la revolución verde en América Latina han afectado la dimensión económica, social y la soberanía alimentaria; y se evidencian en la concentración de la propiedad de la tierra, el empobrecimiento de los pequeños productores quienes no pueden acceder a la maquinaria e insumos, quienes además siguen siendo expropiados y despojados, y a quienes en algunos casos se les ofrece empleo como asalariados, pero no pueden producir alimentos para autoconsumo y para el mercado local. 


\section{Refugiados climáticos}

En el mundo 50 millones de campesinos por año son expulsados de las zonas rurales (Houtart, 2016). Las estadísticas oficiales predicen que los agricultores más pobres son especialmente vulnerables a los impactos del cambio climático debido a su exposición geográfica, bajos ingresos, mayor dependencia en la agricultura para su supervivencia y su limitada capacidad de buscar otras alternativas de vida (Nicholls, 2013). Los migrantes climáticos se están convirtiendo rápidamente en el rostro humano del cambio climático. Según un estudio del Banco Mundial sobre el África subsahariana, el sur de Asia y América Latina (que en conjunto contiene el 55\% de la población de los países en desarrollo), más de 140 millones de personas en estas regiones podrían ser desplazadas internamente para el 2050, sin tomar en cuenta los conflictos armados (Rigaud et al., 2018).

Se estima que, para el 2050, habrá:

- 86 millones de migrantes climáticos internos en África subsahariana.

- 40 millones de migrantes climáticos internos en el sur de Asia.

- 17 millones de migrantes climáticos internos en América Latina.

Este modelo agrícola ha generado una crisis profunda al reducir la complejidad de la agricultura a modelos estándares de monocultivos funcionales a la acumulación del capital del agronegocio. En el contexto actual no se puede negar que el aumento de las emisiones de $\mathrm{CO}_{2} \mathrm{y}$, por ende, el aumento de la temperatura en el planeta está cambiando los patrones climáticos, o lo que se ha llamado eventos extremos. Estos cambios están provocando que la agricultura sea más vulnerable; es decir, que la programación de siembras o de cosechas se vuelva más impredecibles, tornando más inestable la posibilidad de los agricultores de tener una economía que le permita continuar su actividad o conservar sus modos de vida. 
En la Estrategia Nacional de Cambio Climático del Ecuador del año 2012, se reconoce a la Soberanía alimentaria, agricultura, ganadería, acuacultura y pesca como sectores prioritarios de atención debido a que la producción agropecuaria es muy vulnerable a los efectos del cambio climático. El IPCC (2007) ha advertido que la agricultura en latitudes medias será la más afectada por la cada vez menor disponibilidad de agua, de igual forma en los trópicos secos debido a la alteración de las precipitaciones de lluvia y de la evapotranspiración; y en áreas dependientes de la nieve y del deshielo. Estas alteraciones de los patrones climáticos afectan sobre la producción de alimentos y las repercusiones que ello tiene sobre los precios, el acceso de las poblaciones a los productos (MAE, 2019); y, en última instancia, la pérdida del control soberano de sus modos de vida (modos de producir y modos de alimentarse) promueve y profundiza las corrientes migratorias desde el campo hacia la ciudad. Como es evidente, los cambios a nivel global se expresan a nivel, en un estudio denominado "Análisis situacional de la Soberanía Alimentaria en el contexto de la adaptación al cambio climático en el Ecuador", se menciona:

La expansión de monocultivos, con el consecuente uso de plaguicidas y fertilizantes, y la expansión de la agricultura para exportación como banano, café, cacao, flores, abacá, tabaco, frutas tropicales y brócoli, ponen en riesgo la seguridad y soberanía alimentaria, puesto que el interés del pequeño agricultor por ser parte de las cadenas de producción supone un abandono de los multicultivos y de los cultivos de autoconsumo, incrementando su vulnerabilidad ante el cambio climático y afectando su entorno inmediato por pérdida de la diversidad genética, la reducción de la resiliencia de los ecosistemas y el alto uso de químicos. (Cordero, Vásquez, \& Rosero, 2011)

Se ve con claridad que la expansión de monocultivos, la artificialización de los sistemas de producción bajo el paquete tecnológico de la Revolución Verde en el Ecuador está destruyendo el control soberano de la alimentación, deteriorando los modos de vida de los campesinos, la salud ambiental y humana. 


\section{Agroecología y resiliencia al cambio climático}

Numerosas experiencias de la agricultura campesina han demostrado que sus sistemas biodiversos responden muy bien ante las condiciones ambientales cambiantes; sin embargo, como lo expresa Nicholls (2013), la resiliencia ecológica de los agroecosistemas está íntimamente ligada a la resiliencia social, que es la habilidad de las comunidades rurales de generar una infraestructura social capaz de soportar shocks externos. Las estrategias de organización social (redes de solidaridad, intercambio de alimentos, etc.) utilizadas por los agricultores para manejar circunstancias difíciles impuestas por tales eventos son un componente clave de la resiliencia.

Es importante tener en cuenta que la agricultura campesina es muy diversa, que es cierto que son grupos vulnerables ante el cambio climático por ejemplo, pero que además vienen preparándose para las variaciones y cambios que han percibido mediante diferentes estrategias para resistir y recuperarse del paso de eventos climáticos como inundaciones, sequías, huracanes, entre otros, basados principalmente en sus técnicas tradicionales como cosecha de agua, variedades de plantas locales resistentes a los cambios de temperatura o de humedad, sistemas de producción diversificados, conservación de suelos, etc.

En contraste al paradigma del modelo agrícola dominante, que está en crisis y que no da una respuesta sustentable al mundo, surge la necesidad de visibilizar un modelo agrícola alternativo que para Nicholls y Altieri (2012), es el paradigma agroecológico, que tiene su base cultural, social y productiva en la racionalidad etnoecológica de la agricultura familiar campesina, fuente fundamental de un legado importante de saber agrícola tradicional, de agrobiodiversidad y de estrategias de soberanía alimentaria. Entiéndase la soberanía alimentaria como una contrapropuesta al marco de la política macroeconómica neoliberal; la soberanía alimentaria clama por el derecho de las naciones y los pueblos a restringir el comercio, si esto fuera necesario para proteger a los agricultores de pequeña escala y otras comunidades rurales marginadas contra el dumping y la competencia desleal. 
Una particularidad del enfoque agroecológico es la valorización del saber tradicional campesino, teniendo en cuenta que en su evolución secular ha logrado alcanzar resiliencia y sostenibilidad con bajos insumos externos o en ausencia de estos, adaptándose a situaciones socioeconómicas y ambientales generalmente adversas y de escasos recursos (Díaz, 2013).

Es por estas razones, que se considera que muchos agricultores en el mundo y no es la excepción en Ecuador, ya han venido practicando la agroecología, incluso si no se denomina como tal; es decir, trabajando desde la lógica de la conservación de la biodiversidad, del cuidado de los recursos naturales y de la salud de su familia o sus comunidades.

En Ecuador, se reconocen avances en los últimos años en materia de educación, salud, obras de infraestructura, y esto gracias a la recuperación de la institucionalidad y por ende inversión pública. A pesar de ciertos avances en algunos ámbitos de la política pública, en el sector agrícola del país aún persiste la visión reduccionista y funcional al gran capital de que a través de más monocultivos y de la idea que más exportaciones lograrán un cambio de matriz productiva que hará crecer la economía del país, que generará puestos de trabajo; pero si se actúa desde la inmediatez no estamos poniendo en la mesa los gigantes costos ocultos ambientales, el drama social que viven los campesinos al verse nuevamente despreciados y explotados por un sistema enajenante que quizás los vuelve unos asalariados, rompiendo con la posibilidad de conservarlos como campesinos y apoyarlos para que tengan una ruralidad de esperanza.

\section{La LORSA como estrategia para mitigar el cambio climático en Ecuador}

Con base en estos argumentos, se puede aseverar que la agricultura promocionada como investigación, desarrollo e innovación $(\mathrm{I}+\mathrm{D}+\mathrm{i})$, controlada por capitales financieros transnacionales, no ha ofrecido ni ofrecerá soluciones a la soberanía alimentaria mundial, ni 
al cambio climático; por lo cual se debe buscar otras alternativas más integrales y centradas en las necesidades y derechos de los seres humanos y de la naturaleza como la agroecología. En Ecuador contamos con la Ley Orgánica del Régimen de Soberanía Alimentaria (LORSA), que es un cuerpo normativo de vanguardia en América Latina, que se debe utilizar como instrumento de lucha en esta disputa por una agricultura familiar, campesina e indígena. En este cuerpo legal se destaca la función social y ambiental del uso y acceso a la tierra (art. 282 de la Constitución y artículo 6 y 7 LORSA), en la función ambiental de la tierra se menciona que deberá contribuir a la conservación de la biodiversidad, la agrobiodiversidad mediante asociatividad de cultivos y el mantenimiento de las funciones ecológicas como la conservación y manejo integral de cuencas hidrográficas, áreas forestales y bosques; como se puede apreciar esta ley se alinea al fomento de agroecosistemas diversos y comprende y promueve una agricultura con una sustentabilidad fuerte.

En la LORSA se encuentran plasmados estos enfoques que priorizan a la agricultura familiar, campesina e indígena y se promueve fundamentalmente el acceso prioritario de estos grupos humanos a la tierra, al uso y aprovechamiento del agua; se excluye la posibilidad de apropiación del material genético bajo varias modalidades de propiedad intelectual y sistemas de patentes, y algo muy importante de esta ley es que posiciona a la agroecología como un modo de producción sustentable, abriendo el paso para una serie de políticas públicas como programas de capacitación, líneas de crédito y mecanismos de comercialización para su desarrollo y fortalecimiento (art. 14 LORSA). Esta Ley de soberanía alimentaria claramente está amparando un paradigma de agricultura de base campesina, que bien podría ser una herramienta poderosa de profundización del modelo agroecológico, y de una reivindicación del ecologismo popular como lo llama Martínez Alier (2011), es decir, todos los aportes socio-ecológicos de parte de los campesinos, por ejemplo, la mitigación al cambio climático y la conservación de los socio-agroecosistemas rurales. Como resistencia frente a la alternativa autoritaria del capitalismo agrícola, han surgido movimientos rurales y urbanos en el Ecuador, dentro de comunidades campesinas, familias urbanas, activistas y academias, que están alcanzando importantes im- 
pactos locales y regionales y que de forma colectiva se podría potenciar su accionar al usar estos instrumentos normativos como herramientas políticas y de acción transformadora y de esta forma poder ofrecer alternativas de mayor escala ante la crisis alimentaria y ambiental.

\section{Conclusiones}

La soberanía alimentaria es una categoría conceptual y política, es conceptual porque por un lado permite comprender la complejidad de la realidad de los sistemas agroalimentarios; y por otro lado es política, porque disputa a la agricultura dominante o agronegocio el control de la agricultura (agua, tierra, sistemas de comercialización, economía local, etc.). Esta categoría de la soberanía alimentaria constituye el espíritu de la LORSA, y propone a la agroecología como un paradigma alternativo de agricultura donde se respete la reproducción social del campesinado y los agroecosistemas, que en palabras simples permitan la prosperidad de los agricultores y de la naturaleza (ríos, acequias, biodiversidad). La agroecología de base campesina, permite aprender de las experiencias locales de fincas agro-biodiversas llevadas por campesinos y campesinas, pues ellos hacen una agricultura sustentable que puede responder a la crisis agrícola actual y al cambio climático que va a un ritmo acelerado. Estos territorios agroecológicos son espacios de adaptación y resiliencia ante el calentamiento global y a pesar de los aportes sociales, ambientales y ecológicos han estado invisibilizados y abandonados históricamente por los gobiernos de turno, por esto, es urgente una apuesta política por la agroecología a nivel nacional para poder llegar a recuperar los sistemas agroalimentarios que sean solidarios con los campesinos, sustentables en la forma de producir, que se sepa de dónde vienen los alimentos, asegurando procesos de trazabilidad y bioseguridad y, que respeten la soberanía alimentaria de nuestros pueblos.

La LORSA es un instrumento legal que tiene elementos estructurales para la transformación del agro ecuatoriano, y que debería permitir fortalecer la lucha del movimiento campesino y de los mo- 
vimientos urbanos de consumo consciente. Pero que se evidencia que la visión productivista tecnoburocrática de los gobiernos de turno amalgamadas con los intereses del agronegocio no han permitido que se avance con una propuesta como la LORSA. La arena legislativa, es una arista importante desde donde se puede empujar e impulsar las políticas públicas, si las fuerzas sociales llevaron al nacimiento de la LORSA, es necesario continuar con ese proceso de lucha para que el estado cumpla lo que el pueblo demanda, y lo que demanda el pueblo son relaciones más justas y respetuosas de esta sociedad con su ambiente, con sus campesinos, con la salud colectiva, que el pueblo ecuatoriano no pierda la autonomía de poder alimentarse en forma diversa y nutritiva, no perder la autonomía de sus decisiones de alimentarse con productos sin agrotóxicos, la autonomía de decidir qué tipo de sociedad quiere construir, donde todos de forma solidaria y consciente podamos hacer prosperar el trabajo de nuestros campesinos.

\section{Bibliografía}

Cordero, V., Vásquez, P., \& Rosero, C. (2011). Análisis situacional de la soberanía alimentaria en el contexto de la adaptación al cambio climático en el Ecuador. En Estrategia Nacional de Cambio Climático del Ecuador 2012-2025. Quito, Ecuador. Recuperado de: https:// bit.ly/2SAFxo8

Díaz, B. (2013). Cambio climático, agricultura y soberanía alimentaria: transnacionales versus agroecología. La transformación agroecológica de Cuba. Buenos Aires: CLACSO.

Fanelli, D. (2007). Meat is murder on the environment. The Scientist, 195(2613), 15. https://doi.org/10.1016/s0262-4079(07)61804-1.

FAO (2011). World Livestock 2011. Livestock in food security. A. McLeod (Ed.). Roma: FAO.

(2012). Sustainability Pathways. Livestock and Landscapes. Livestock and Landscapes, All.

(2015). The state of food and agriculture. https://doi.org/10.18356/79 dbc75b-en.

Food and Agriculture Organization of the United Nations (FAO) (2013). Food wastage footprint. Food and Agriculture Organization of the United Nations (FAO).

George, S. (1981). Les Stratèges de la faim. Ginebra. 
Gerber, P.J., Steinfeld, H., Henderson, B., Mottet, A., Opio, C., Dijkman, J., Falcucci, A. \& Tempio, G. (2013). Tackling climate change through livestock. A global assessment of emissions and mitigation opportunities. Vol. 14. Roma: Food and Agriculture Organization of the United Nations (FAO).

Houtart, F. (2016). Las agriculturas familiares campesinas e indígenas como base de una reforma agraria popular en Ecuador. En 50 años de Reforma Agraria. Cuestiones pendientes y miradas alternativas. Primera ed. Quito: Universidad Andina Simón Bolívar.

IPCC (Intergovernmental Panel on Climate Change) (2007). Cambio climático 2007: Informe de síntesis. Contribución de los Grupos de trabajo I, II y III al Cuarto Informe de evaluación del Grupo Intergubernamental de Expertos sobre el Cambio Climático. Ginebra, Suiza. Recuperado de: https://bit.ly/39Gbm4p

(2014). II y III al Quinto Informe de Evaluación del Grupo Contribución de los Grupos de trabajo I, Intergubernamental de Expertos sobre el Cambio Climático. Cambio climático 2014 - Informe de síntesis. Editado por R.K. Meyer, Pachauri, y L.A. Ginebra, Suiza: IPCC. MAE (2019). Primera contribución determinada a nivel nacional para el Acuerdo de París bajo la Convención Marco de Naciones Unidas sobre Cambio Climático.

Martínez Alier, J. (2011). El ecologismo de los pobres: Conflictos ambientales $y$ lenguajes de valoración. Quinta ed. Barcelona: Icaria Editorial s.a.

McMichael, P. (2012). The land Grab and Corporate Food Regime Restructuring. The Journal of peasant Studies, 39, 681-701.

Nicholls, C. (2013). Enfoques agroecológicos para incrementar la resiliencia de los sistemas agrícolas al cambio climático. En REDAGRES, CYTED, SOCLA (Ed.), Agroecología y resiliencia socioecológica: adaptándose al cambio climático. Medellín, Colombia.

Nicholls, C., \& Altieri, M. (2012). Modelos ecológicos y resilientes de producción agrícola para el siglo XXI. Agroecología, 6(0), 28-37.

Rigaud, K., de Sherbinin, A., Jones, B., Bergmann, J., Clement, V., Ober, K... et al. (2018). Groundswell: Preparing for Internal Climate Migration. Washington, DC: The World Bank, 256. https://doi.org/doi. org/10.7916/D8Z33FNS.

Rubio, B. (2010). De agrocombustibles y expansión minera en América Latina. Revista Territorios, 5, 15-36.

(2015). El dominio del hambre: crisis de hegemonía y alimentos. Editado por Universidad Autónoma de Chapingo-Colegio de Postgraduados: Universidad Autónoma de Zacatecas: Juan Pablos Editor. Segunda Ed. México. 